\title{
Correlation of Serum C-Reactive Protein with Disease Severity in Tuberculosis Patients
}

\author{
Mohammad Shameem $^{1 *}$, Nazish Fatima ${ }^{2}$, Asrar Ahmad ${ }^{1,3}$, Abida Malik $^{2}$, Qayyum Husain $^{3}$ \\ ${ }^{1}$ Department of Tuberculosis and Chest Diseases, Jawaharlal Nehru Medical College, Aligarh Muslim University, Aligarh, India \\ ${ }^{2}$ Department of Microbiology, Jawaharlal Nehru Medical College, Aligarh Muslim University, Aligarh, India \\ ${ }^{3}$ Department of Biochemistry, Faculty of Life Sciences, Aligarh Muslim University, Aligarh, India \\ Email: *drshameem123@gmail.com
}

Received June 13, 2012; revised July 25, 2012; accepted August 6, 2012

\begin{abstract}
Purpose: To study the factors influencing sputum smear conversion including Serum C-Reactive Protein (CRP) and its correlation with disease severity in tuberculosis patients. Method: Levels of Serum-CRP concentrations were determined in 60 patients with pulmonary tuberculosis, 30 healthy volunteers and patients in follow-up after completion of antitubercular treatment (DOTS therapy). Results: Serum-CRP levels were found to be significantly higher in smear-positive group as compared with the follow-up patients and smear-negative control group. The values were 43.65 $\pm 23.68,9.88 \pm 5.23$ and $4.04 \pm 3.85 \mathrm{mg} / \mathrm{L}$ respectively $(\mathrm{P}<0.0001)$. Among the smear-positive patients, Serum-CRP levels were the highest in AFB3+ patients (65.28 \pm 10.32$)$ as compared with the AFB2+ patients (35.93 \pm 7.22$)$, AFB1+ patients $(16.37 \pm 2.62)$ and AFB scanty patients $(10.92 \pm 2.97)$ respectively, the difference was found statistically significant $(\mathrm{P}<0.0001)$. Correlation of predictors of sputum smear conversion also revealed that these values were significantly higher in active pulmonary tubercular patients as compared to control. It is also found significant positive correlation between AFB smear positive patients with levels of Serum-CRP concentration. Conclusion: Serum-CRP levels are significantly correlated with disease severity in patients with active pulmonary tuberculosis. Thus these findings from the present study would certainly add new criteria for early diagnosis of TB, which may lead to development of new strategies to treat TB.
\end{abstract}

Keywords: Acid Fast Bacilli; Serum C-Reactive Protein; Tuberculosis; Directly Observed Therapy Short-Course

\section{Introduction}

Tuberculosis (TB) is a socio-economic as well as highly contagious disaster that is occurring worldwide, especially in Asia and Africa. It is one of the most serious infectious diseases and a considerable public health problem due to its high risk of person-to-person transmission, morbidity, and mortality. World Health Organization (WHO) report 2010 stated that more than 2 billion people, equal to one-third of the world's population, are infected with TB bacilli, the microbes that cause TB. There were estimated 9.4 million new TB cases worldwide in 2008 including 1.4 million cases among people living with HIV of which 1.8 million people died from TB. However, India has the highest incidence of tuberculosis cases in the world. It accounts for more than $1 / 5$ th of the global TB cases. As per current estimate of WHO, 2.4 percent untreated TB patients and 14.7 percent previously treated patients had multi-drug resistant tuberculosis (MDR-TB). Approximately 500,000 cases of MDRTB emerge every year globally. Of this, India and China

${ }^{*}$ Corresponding author. account for more than 50 percent cases [1].

Despite the availability of effective diagnostic, preventive, and curative technologies, TB remains the number one cause of adult deaths by a curable infectious disease worldwide. The main source of infection is the patient with active pulmonary $\mathrm{TB}$ and positive sputum smears who excretes tubercle bacilli [2]. Factors associated with infectivity include bacillary load, severity of coughing, proximity to the patient and the duration of anti-TB therapy. In addition, various serological markers have been evaluated as indicators of disease activity, but their clinical usefulness remains uncertain. The most widely accepted measure of treatment response in patients with pulmonary TB is the disappearance of acid fast bacilli (AFB) from both sputum smear and culture [3].

After the start of treatment, the number of bacilli in the sputum decreases rapidly and in more than 90 percent of cases sputum smears become negative for AFB at 3 months. Studies have also shown that the time for sputum sterilization (indicated by sputum negativity at 2 or 3 months) is an important determinant of relapse [4-8]. 
New risk factors for TB, such as human immunodeficiency virus (HIV) infection are challenging problems for TB control all over the world. HIV infected patients with TB have more risk for treatment failure than HIV uninfected patients $[9,10]$.

Serum C-Reactive Protein (CRP) was first reported in 1930 in human blood of those with acute inflammatory diseases by Tillet and Francis [11]. Serum-CRP is an acute phase reactant synthesized by hepatocytes under the influence of interleukin-1 arising at sites of infection, inflammation and trauma, and thought to participate in handling of abnormal extrinsic and autologous materials $[12,13]$. Human Serum-CRP is a member of the class of acute phase reactants as its levels rise during inflammatory processes occurring in the body. Human SerumCRP is thought to assist in complement binding to foreign and damaged cells and affect the humoral response to disease. Human Serum-CRP is also believed to play an important role in innate immunity, as an early defense system against infections.

The aim of this study is to evaluate the concentration of Serum-CRP in pulmonary tuberculosis as a predictor of sputum smear conversion and to see correlation between Serum-CRP and disease severity.

\section{Materials and Methods}

This study was conducted in Department of Tuberculosis and Respiratory Diseases, Jawaharlal Nehru Medical College Hospital, Aligarh Muslim University, Aligarh, on patients attending Out Patient Department (OPD) and In Patient Departments (IPD) of the College Hospital from $1^{\text {st }}$ April 2008 to $31^{\text {st }}$ May 2010. We documented 60 Sputum Positive pulmonary tubercular cases (45 male and 15 female respectively) and 30 Healthy Control (22 male and 8 female respectively). Factors (including Serum-CRP) associated with sputum smear conversions were investigated.

\subsection{Study Population}

The study group consisted of cases of active pulmonary tuberculosis diagnosed on the basis of persistent clinical signs and symptoms with positive sputum smear (at least one sputum-smear found AFB positive out of three consecutive specimens). All cases were negative for HIV tests except one. Each patient's demographic profile, clinical, radiological findings, co-morbid disease, sputum smear examination; drug susceptibility pattern and treatment details were recorded. Both sputum smear and culture conversion and Serum-CRP levels were determined. The diagnosis of TB as made with the documentation of AFB through Ziehl-Neelsen (ZN) staining of sputum and the isolation of $M$. tuberculosis in sputum culture on Lowenstein Jensen (LJ) solid medium. First line anti-TB therapy was started when the first positive smear was detected. Patients were treated according to the guidelines of the Revised National Tuberculosis Control Programme (RNTCP) as isoniazid $(\mathrm{H})$, rifampicin (R), pyrazinamide $(Z)$, ethambutol $(E)$ during the initial phase and $H$ plus $R$ during the continuation phase. Patients took their drugs regularly in the DOTS and were analyzed for at least 1 month, or until sputum AFB smears were negative for three consecutive days. Sputum smear test was performed monthly in a routine programme. Social status of patients was assessed by per capita monthly income divided by family member dependent on that income [14]. Smoking History of patients was calculated by this given formula

$$
\text { Number of pack years }=\frac{\text { No. of cigarette or bidi smoked/day } \times \text { No. of years smoked }}{20}
$$

\subsection{Collection of Specimen}

The patients were advised to collect the early morning sputum specimen (about $4-5 \mathrm{ml}$ ) collected in a wide mouthed sterile plastic vial having lid with safety lock and was transported to Mycobacteria laboratory for further processing. They were instructed to rinse their mouth with pure water and clean their teeth (without using tooth paste or disinfectant) before collection to avoid contamination with food and other particles and were instructed to stand facing a wall, away from wind and keep both hands on hips and to cough forcibly to bring out material from the lungs and not merely saliva. Sputum specimens were obtained from each patient at the time of $1^{\text {st }}$ visit for AFB smear and Serum-CRP concen- tration as well as at the time after treatment completed in follow-up.

After a detailed history and physical examination, the $5 \mathrm{ml}$. venous blood sample was drawn into tubes and kept at room temperature for one hour or until it clotted. After that the samples were centrifuged at $1500 \mathrm{rpm}$ for 5 minutes and clot was broken from side wall of tub

e and again centrifuged at $1500 \mathrm{rpm}$ for 5 minutes to separate the serum in a storage vial, which was then stored at $-20^{\circ} \mathrm{C}$.

The sputum samples were examined for AFB by direct microscopic examination. It was carried out after concentration and decontamination of specimen and grading of Ziehl-Neelson stained smears was carried out as per the standard procedure. The counting of the number of 
bacilli in each smear was done according to revised RNTCP grading. Modified Lowenstein-Jensen medium was described by the International Union against tuberculosis and used for the primary isolation of the strains of Mycobacteria.

The hsCRP ELISA (Biotron Diagnostics Inc, Hemet, California, USA) was used for estimation of Serum-CRP. It is based on the principle of a solid phase enzymelinked immunosorbent assay [15]. The assay system utilizes a unique monoclonal antibody directed against a distinct antigenic determinant on the CRP molecules. The test sample is allowed to react simultaneously with the two antibodies, resulting in the CRP molecules being sandwiched between the solid phase and enzyme-linked antibodies.

Written informed consent was obtained from each recruited subjects and the Institutional Ethics Committee of the Medical College approved the study.

\subsection{Statistical Analysis of Data}

Statistical analyses were performed with SPSS for Windows (Version 16.0; SPSS Inc.) and Graph Pad Prism 5.01. Data are expressed as mean $\pm \mathrm{SD}$. Analysis of the data was done using one-way ANOVA, unpaired student t-test and Fisher's exact test. $\mathrm{P}$ value $<0.05$ was considered significant.

\section{Results}

There were 60 active pulmonary tubercular patients (45 male and 15 female) documented with mean age $34.17 \pm$ 15.07 (13 - 80 years). They were sputum smear positive patients and had lower middle and poor socio-economic status with low BMI. The characteristics of the patients and Healthy Control are listed in Table 1. Among the sputum smear positive patients, 3 patients were found to be scanty, 16 were AFB1+, 12 were AFB2+ and the remaining 29 were AFB3+. Serum-CRP levels were estimated in all of these active tubercular patients before treatment and after treatment as well as in control. The levels of Serum-CRP concentration were also analyzed in correlation with AFB smear. Serum-CRP levels were found to be significantly higher in smear-positive group as compared with the follow-up patients (after treatment) and smear-negative control group. Figure 1 clearly shows that the Serum-CRP levels were significantly higher in sputum-positive patients when compared to smear-negative group. The values were $43.65 \pm 23.68$, $9.88 \pm 5.23$ and $4.04 \pm 3.85 \mathrm{mg} / \mathrm{L}$ respectively $(\mathrm{P}<$ $0.001)$.

Among the smear-positive patients, Serum-CRP levels were the highest in AFB3+ patients $(65.28 \pm 10.32)$ as compared with the AFB2+ patients $(35.93 \pm 7.22)$, AFB1+ patients $(16.37 \pm 2.62)$ and AFB scanty patients

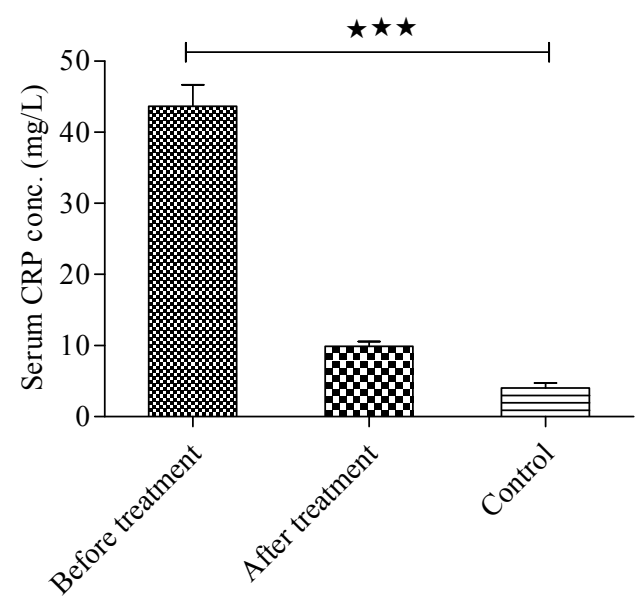

Figure 1. Relation between Serum-CRP conc. of pulmonary tubercular patients before and after treatment (6 months) with Healthy Control ${ }^{* * *} \mathrm{P}<\mathbf{0 . 0 0 1}$.

$(10.92 \pm 2.97)$ respectively, the difference was found statistically significant $(\mathrm{P}<0.001)$ (Figure 2). Table 2 shows the correlation between predictors of sputum conversion and Serum-CRP in tubercular patients before treatment.

Pearson's correlation coefficients of different predicttors of sputum conversion with Serum-CRP in tubercular patients before treatment are presented in Table 2. It showed significant negative correlation between BMI and social status with Serum-CRP in TB patients. It also showed significant positive correlation between AFB smear positive with Serum-CRP.

Figure 3 shows the high Serum-CRP conc. in AFB+ ve patients compared to AFB- ve patients after treatment. Patient with high Serum-CRP conc. at base line took longer time of sputum conversion and 5 patients remained $\mathrm{AFB}+$ ve after treatment (6 months).

\section{Discussion and Conclusions}

Serum-CRP is one of the most common acute phase reactant proteins used as an indicator of inflammation. Now it has attracted the attention of various researchers, as an indicator to differentiate tubercular from non-tubercular infections or diagnose extra pulmonary tuberculosis. However, reports have been conflicting. One study found out that elevated serum levels of Serum-CRP can be useful in differentiating tuberculosis from non-tubercular diseases [16], whereas another study found it to be of not much value [17]. Moreover, few studies which have found it to be useful were in patients of pleural effusion [18].

We extrapolated this observation and hypothesized that even if Serum-CRP levels are not unequivocal in differentiating tubercular from non-tubercular infections, still these levels could be useful as a marker of extent or severity of disease. 
Table 1. Demographic profile of patients with active pulmonary tuberculosis and healthy controls.

\begin{tabular}{|c|c|c|c|c|}
\hline Variable & TB Patients & Healthy Control & OR (95\% CI) & P Value \\
\hline & $\mathrm{n}(\%)$ & $\mathrm{n}(\%)$ & & \\
\hline \multicolumn{5}{|l|}{ Gender } \\
\hline Male & $45(75)$ & $22(73.33)$ & $1.09(0.40-2.96)$ & $1.00^{\mathrm{ns}}$ \\
\hline Female & $15(25)$ & $8(26.67)$ & & \\
\hline Age & $34.17 \pm 15.07$ & $33.43 \pm 12.94$ & & \\
\hline $0-20$ & $16(26.67)$ & $2(6.67)$ & $7.36(1.52-35.58)$ & $0.009^{* *}$ \\
\hline $21-40$ & $25(41.66)$ & $23(76.67)$ & & \\
\hline $41-60$ & $16(26.67)$ & $4(13.33)$ & $1.33(0.11-16.49)$ & $1.00^{\mathrm{ns}}$ \\
\hline$>60$ & $3(5)$ & $1(3.33)$ & & \\
\hline BMI & $16.75 \pm 2.59$ & $21.36 \pm 2.78$ & & \\
\hline$<18.5\left(\mathrm{Kg} / \mathrm{m}^{2}\right)$ & $44(73.33)$ & $5(16.67)$ & $13.75(4.50-42.06)$ & $0.0001^{* * *}$ \\
\hline$\geq 18.5\left(\mathrm{Kg} / \mathrm{m}^{2}\right)$ & $16(26.67)$ & $25(83.33)$ & & \\
\hline \multicolumn{5}{|l|}{ Co-Morbidities } \\
\hline Yes & $13(21.67)$ & $11(36.67)$ & $0.4778(0.18-1.25)$ & $0.14^{\mathrm{ns}}$ \\
\hline No & $47(78.33)$ & $19(63.33)$ & & \\
\hline \multicolumn{5}{|c|}{ Drug Abuse/Alcoholics } \\
\hline Yes & $10(16.67)$ & $2(6.67)$ & $2.8(0.57-13.70)$ & $0.32^{\mathrm{ns}}$ \\
\hline No & $50(83.33)$ & $28(93.33)$ & & \\
\hline \multicolumn{5}{|l|}{ Food Habit } \\
\hline Vagetarian & $12(20)$ & $5(16.67)$ & $1.25(0.40-3.95)$ & $0.78^{\mathrm{ns}}$ \\
\hline Non-Veg & $48(80)$ & $25(83.33)$ & & \\
\hline \multicolumn{5}{|l|}{ Social Status } \\
\hline Very Poor & $3(5)$ & $2(6.67)$ & $0.17(0.02-1.50)$ & $0.15^{\mathrm{ns}}$ \\
\hline Poor & $26(43.34)$ & $3(10)$ & & \\
\hline Lower Middle & $23(38.33)$ & $11(36.67)$ & $4.18(1.15-15.22)$ & $0.03^{*}$ \\
\hline Upper Middle & $5(8.33)$ & $10(33.33)$ & & \\
\hline High & $3(5)$ & $2(6.67)$ & $7(0.22-219.1)$ & $0.43^{\mathrm{ns}}$ \\
\hline Upper High & 0 & $2(6.67)$ & & \\
\hline Smoking History & $3.5 \pm 5.98$ & $0.63 \pm 1.3$ & & \\
\hline Yes & $22(36.67)$ & $7(23.33)$ & $1.90(0.70-5.15)$ & $0.23^{\mathrm{ns}}$ \\
\hline No & $38(63.33)$ & $23(76.67)$ & & \\
\hline Serum CRP & $18.83 \pm 7.87$ & $4.035 \pm 3.85$ & & \\
\hline$<15(\mathrm{mg} / \mathrm{L})$ & $11(18.33)$ & $29(96.67)$ & $0.01(0.001-0.06)$ & $<0.0001^{* * *}$ \\
\hline$\geq 15(\mathrm{mg} / \mathrm{L})$ & 49 (81.67) & $1(3.33)$ & & \\
\hline \multicolumn{5}{|l|}{ X-Ray Findings } \\
\hline Cavitary & $40(66.67)$ & $4(13.33)$ & $13(3.99-42.39)$ & $<0.0001^{* * *}$ \\
\hline Non-Cavitary & $20(33.33)$ & $26(86.67)$ & & \\
\hline
\end{tabular}

OR-odds ratio; CI-confidence interval; ${ }^{\mathrm{ns}}>0.05 ;{ }^{*} \mathrm{P}<0.05 ;{ }^{* *} \mathrm{P}<0.01 ;{ }^{* * *} \mathrm{P}<0.001$ Results were expressed as total no. (\%) and mean $\pm \mathrm{SD}$. 
Table 2. Coefficients of correlation between predictors of sputum conversion and Serum-CRP in tubercular patients before treatment.

\begin{tabular}{ccc}
\hline Variables & $\begin{array}{c}\text { Correlation } \\
\text { Coefficients (r) }\end{array}$ & P Value \\
Age & 0.17 & $0.20^{\mathrm{ns}}$ \\
Sex & 0.16 & $0.22^{\mathrm{ns}}$ \\
BMI & -0.29 & $0.03^{*}$ \\
Co-Morbidities & -0.19 & $0.16^{\mathrm{ns}}$ \\
Drug Abuse/Alcoholics & 0.11 & $0.39^{\mathrm{ns}}$ \\
Food Habit & -0.15 & $0.25^{\mathrm{ns}}$ \\
Social Status & -0.32 & $0.01^{*}$ \\
Smoking History & 0.22 & $0.09^{\mathrm{ns}}$ \\
X-Ray Findings & -0.18 & $0.18^{\mathrm{ns}}$ \\
AFB Smear Positivity & 0.92 & $0.000^{* * *}$ \\
\hline
\end{tabular}

${ }^{\mathrm{ns}} \mathrm{P}>0.05 ;{ }^{*} \mathrm{P}<0.05 ;{ }^{* * *} \mathrm{P}<0.001$.

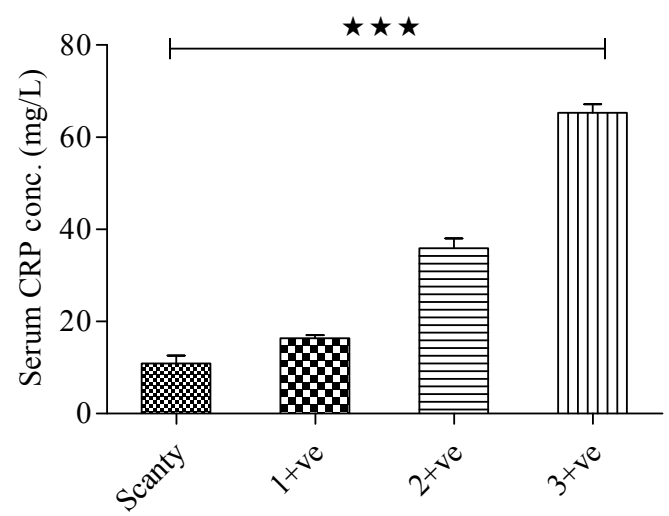

Figure 2. Relation between Serum-CRP conc. among patients with active pulmonary tuberculosis according to AFB smear grading. ${ }^{* * *} \mathbf{P}<\mathbf{0 . 0 0 1}$.

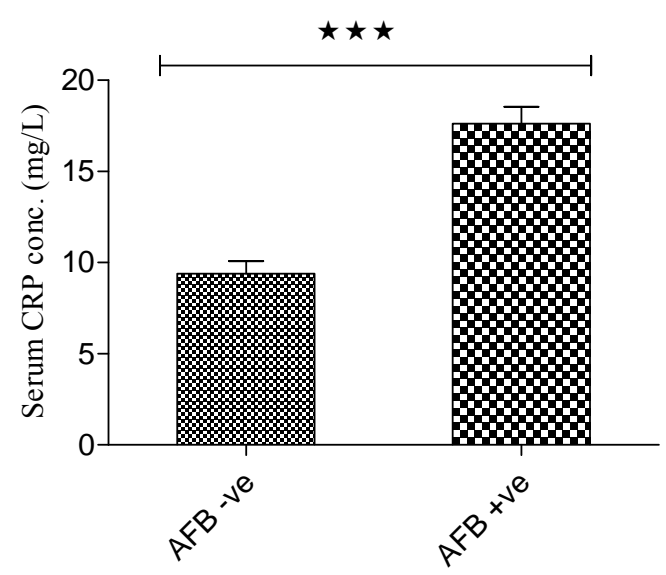

Figure 3. Serum-CRP conc. in patients with AFB- ve and AFB+ ve patients after 6 month of DOTS therapy. ${ }^{* * *} \mathbf{P}<$ 0.001.
It is very interesting to note that sputum conversion and disease improvement are associated with SerumCRP level. All patients have high Serum-CRP concentration than Healthy Control. The follow-up patients have high BMI and low Serum-CRP conc. than active pulmonary tubercular patients at initial stage. The patients were also compared with Healthy Control and have high Serum-CRP concentration with low BMI and poor socioeconomic status. AFB3+ patients also have high SerumCRP concentration than AFB2+, AFB1+ and AFB scanty patients.

Patients with low BMI, Co-morbid disease (Diabetes mellitus/COPD/Asthma) and smoking history have high Serum-CRP concentration than the other groups. It also shows that patients with old age, male sex, smoking history, poor socio-economic status and drug abuse/ alcoholics were found to be significantly associated with Serum-CRP concentration and AFB positivity. In the statistic analysis, the presence of co-morbid conditions and low BMI, smoking history with high Serum-CRP concentration were determined as independent factors associated with persistent sputum smear positivity at the end of antitubercular treatment. Pulmonary destruction as evidenced by tuberculous cavitation signifies severe disease and thus CRP values of the present patients with cavitation were higher than those without. Baynes, et al., found that Serum-CRP concentrations in patients with pulmonary tuberculosis only gradually normalised over several weeks during antitubercular (DOTS) therapy [19]. Serial Serum-CRP measurements have been used in monitoring the efficacy of diagnostic chemotherapy in patients with only radiological suspicion of tuberculosis [20]. However, modern antituberculosis chemotherapy includes rifampicin, a broad spectrum antibiotic. Thus bacterial infection other than tuberculosis may also be cured, invalidating the diagnostic significance of SerumCRP measurements. This study also showed that the high Serum-CRP conc. took longer time of sputum conversion than the lower conc. of Serum-CRP. Out of 60 patients, 5 patients remained sputum positive after treatment and they contain high Serum-CRP conc. It also indicates the prognosis value of MDR.

Thus, in the present study it was found that the tuberculosis patients displayed high prevalence of Chronic Energy Deficiency as compared to the healthy controls and the findings likely to emerge from the present study would certainly add new knowledge in TB research, which may lead to development of new strategies to treat TB. Keeping in view the rising incidence of TB and a large proportion of world population infected with TB, the development of more effective strategy would be of considerable importance.

The present study also demonstrated that the Serum-CRP levels differ significantly between patients 
grouped according to severity. Thus Serum-CRP level have led to better insight in the understanding of disease severity which plays an important role in the early diagnosis and clinical outcome of the disease. Present study shows that nutritional status as assessed by various anthropometric indices of the TB patients significantly improved with treatment.

\section{Acknowledgements}

The project was sanctioned by Council of Science and Technology (CST-UP), Lucknow UP. The authors are thankful to CST-UP for the grant.

\section{REFERENCES}

[1] "WHO Global Tuberculosis Control Report 2010. Summary," Central European Journal of Public Health, Vol. 18, No. 4, 2010, p. 237.

[2] A. Domínguez-Castellano, M. A. Muniain, J. RodriguezBaño, M. Garcia, M. J. Rios, J. Galvez and R. Perez-Cano, "Factors Associated with Time to Sputum Smear Conversion in Active Pulmonary Tuberculosis," The International Journal of Tuberculosis and Lung Disease, Vol. 7, No. 5, 2003, pp. 432-438.

[3] M. D. Epstein, N. W. Schluger and A. L. Davidow, "Time to Detection of Mycobacterium Tuberculosis in Sputum Culture Correlates with Outcome in Patients Receiving Treatment for Pulmonary Tuberculosis," Chest, Vol. 113, No. 2, 1998, pp. 379-386. doi:10.1378/chest.113.2.379

[4] R. Vidal, N. Martín-Casabona and A. Juan, "Incidence and Significance of Acid-Fast Bacilli in Sputum Smears at the End of Antituberculous Treatment," Chest, Vol. 109, No. 6, 1996, 1562-1565. doi:10.1378/chest.109.6.1562

[5] R. Singla, M. M. Osman and N. Khan, "Factors Predicting Persistent Sputum Smear Positivity among Pulmonary Tuberculosis Patients 2 Months after Treatment," The International Journal of Tuberculosis and Lung Disease, Vol. 7, No. 1, 2003, pp. 58-64.

[6] D. A. Mitchison, “Assessment of New Sterilizing Drugs for Treating Pulmonary Tuberculosis by Culture at 2 Months," The American Review of Respiratory Disease, Vol. 147, No. 4, 1993, pp. 1062-1063.

[7] L. P. Ormerod, O. R. McCarthy, R. M. Rudd and N. Horsfield, "Short Course Chemotherapy for Pulmonary Tuberculosis," Respiratory Medicine, Vol. 85, No. 4, 1991, pp. 291-294. doi:10.1016/S0954-6111(06)80099-5

[8] F. C. Warring Jr. and U. Sutramongkole, "Nonculturable Acid-Fast Forms in the Sputum of Patients with Tuberculosis and Chronic Pulmonary Disease," The American Review of Respiratory Disease, Vol. 102, No. 5, 1970, pp. 714-724.

[9] Z. Liu, K. L. Shilkret and H. M. Ellis, "Predictors of Sputum Culture Conversion among Patients with Tuberculosis in the Era of Tuberculosis Resurgence," Archives of Internal Medicine, Vol. 159, No. 10, 1999, pp. 11101116. doi:10.1001/archinte.159.10.1110

[10] D. R. Burwen, A. B. Bloch, L. D. Griffin, et al., "National Trends in the Concurrence of Tuberculosis and Acquired Immunodeficiency Syndrome," Archives of Internal Medicine," Vol. 155, No. 12, 1995, pp. 1281-1286. doi:10.1001/archinte.1995.00430120062008

[11] W. S. Tillet, W. F. Goebel and O. T. Avery, "Chemical and Immunological Properties of a Species Specific Carbohydrate of Pneumococci," The Journal of Experimental Medicine, Vol. 52, No. 6, 1930, pp. 895-900. doi:10.1084/jem.52.6.895

[12] H. Peltola, M. L. Laipio and M. A. Siimes, "Quantitative C-Reactive Protein (CRP) Determined by an Immunoturbidimetric Method in Rapid Differential Diagnosis of Acute Bacterial and Viral Diseases of Children," Acta Paediatrica Scandinavica, Vol. 73, No. 2, 1984, pp. 273274. doi:10.1111/j.1651-2227.1984.tb09944.x

[13] S. W. Chensue, M. P. Davey, D. G. Remick and S. L. Kunkel, "Release of Interleukin-1 by Peripheral Blood Mononuclear Cells in Patients with Tuberculosis and Active Inflammation," Infection and Immunity, Vol. 52, No. 1, 1986, pp. 341-343.

[14] A. K. Agarwal, "Social Classification: The Need to Update in the Present Scenario," Indian Journal of Community Medicine, Vol. 33, No. 1, 2008, pp. 50-51. doi:10.4103/0970-0218.39245

[15] M. Uotila, E. Ruoslahti and E. Engvall "Two-Site Sandwich Enzyme Immunoassay with Monoclonal Antibodies to Human Alpha-Fetoprotein," Journal of Immunological Methods, Vol. 42, No. 1, 1981, pp. 11-15. doi:10.1016/0022-1759(81)90219-2

[16] C. M. Choi, C. I. Kang, W. K. Jeung, et al., "Role of C-Reactive Protein for the Diagnosis of Tuberculosis among Military Personnel in South Korea," The International Journal of Tuberculosis and Lung Disease, Vol. 11, No. 2, 2007, pp. 233-236.

[17] M. Kannapiran, C. Immanuel, P. V. Krishnamurthy, et al., "C-Reactive Protein Levels in Patients with Pulmonary Tuberculosis,” Lung India, Vol. 7, No. 1, 1989, pp. 34-36.

[18] E. Garcia-Pachon, M. J. Soler, I. Padilla-Navas, et al., "CReactive Protein in Lymphocytic Pleural Effusions: A Diagnostic Aid in Tuberculous Pleuritis," Respiration, Vol. 72, No. 5, 2005, pp. 486-489. doi:10.1159/000087672

[19] R. Baynes, W. Bezwoda, T. Bothwell, Q. Khan and N. Mansoor, "The Non-Immune Inflammatory Response: Serial Changes in Plasma Iron, Iron-Binding Capacity, Lactoferrin, Ferritin and C-Reactive Protein," Scandinavian Journal of Clinical \& Laboratory Investigation, Vol. 46, No. 7, 1986, pp. 695-704. doi: $10.3109 / 00365518609083733$

[20] F. C. de Beer, A. E. Nel, R. P. Gie, P. R. Donald and A. F. Strachan, "Serum Amyloid A Protein and C-Reactive Protein Levels in Pulmonary Tuberculosis: Relationship to Amyloidosis," Thorax, Vol. 39, No. 3, 1984, pp. 196200. doi: $10.1136 /$ thx.39.3.196 